\title{
A limit on the effect of rectified diffusion in volcanic systems
}

\author{
M. Ichihara ${ }^{1}$ and E. E. Brodsky ${ }^{2}$ \\ Received 23 September 2005; revised 8 December 2005; accepted 13 December 2005; published 28 January 2006.
}

[1] Forced oscillations can push dissolved volatiles into bubbles by a process called rectified diffusion. In engineering applications, the pumping action of rectified diffusion makes bubbles grow. In the geosciences, rectified diffusion is a suggested mechanism to trigger volcanic eruptions with seismic waves generated by distant earthquakes. Previous geoscience studies adopted the engineering results and proposed that in a confined system like a magma chamber, rectified diffusion causes pressure increase rather than bubble growth. However, the volcanic application is fundamentally different than engineering applications in that solubility continually changes with increasing pressure in the confined system. Here we present the first self-consistent treatment of rectified diffusion in a confined system. Evolving solubility has a significant effect. The new solution demonstrates that previous work significantly overestimated the effect of rectified diffusion in magmatic systems. For reasonable seismic wave amplitudes, the pressure change is at the most $2 \times 10^{-9}$ of its initial value. Citation: Ichihara, M., and E. E. Brodsky (2006), A limit on the effect of rectified diffusion in volcanic systems, Geophys. Res. Lett., 33, L02316, doi:10.1029/ 2005 GL024753.

\section{Introduction}

[2] When a sound field impinges on a fluid, a bubble may grow due to a mass transport process called rectified diffusion. During the expansion phase of an oscillation, the solubility of volatiles decreases, and in the absence of any other diffusive process, volatiles exsolve into the bubble. During contraction, the opposite effect occurs.

[3] Bubbles take in more volatiles during expansion than they discharge during contraction [e.g., Hsieh and Plesset, 1961; Eller and Flynn, 1965]. The two most significant reasons for the asymmetry are: (1) the interface is larger during expansion than during contraction and (2) radial bubble expansion tangentially stretches the diffusion layer and sharpens the radial gradient of the volatile concentration in the diffusion layer, so that the volatile flux into the bubble is enhanced. This pumping of volatiles into the bubble is known as rectified diffusion.

[4] Sturtevant et al. [1996] and Brodsky et al. [1998] pointed out that rectified diffusion might explain volcanic eruptions triggered by distant earthquakes like those observed by Linde and Sacks [1998]. The earthquakes generate seismic waves and the dilatational strain of the waves transfers volatiles from magma into bubbles by rectified

\footnotetext{
${ }^{1}$ Earthquake Research Institute, University of Tokyo, Tokyo, Japan.

${ }^{2}$ Department of Earth and Space Sciences, University of California, Los Angeles, California, USA.
}

Copyright 2006 by the American Geophysical Union. 0094-8276/06/2005GL024753\$05.00 diffusion. It was argued that this volatile transfer increases the pressure of the system if bubble expansion is restricted in a confined liquid. Brodsky et al. [1998] recognized that the solubility of the volatiles must increase with increasing pressure and proposed that there existed a limit on the pressure increase attainable by rectified diffusion. They used the classic solution of Hsieh and Plesset [1961] to calculate the mass transfer from rectified diffusion and then solved for the limiting pressure of the system. They concluded that the maximum attainable pressure was determined by the degree of supersaturation of the system.

[5] However, the problem of the limiting pressure cannot be decoupled from the rectified diffusion solution in a confined system. Since the most important effect in generating a mass flux during a strain cycle is the change in solubility of volatiles, then changes in pressure (and hence solubility) must be fully coupled in computing the rate of volatile transfer via rectified diffusion. The Hsieh and Plesset [1961] solution assumed that the pressure, bubble radius, and volatile concentration all oscillate around the fixed values. The transient problem of mass transport by rectified diffusion has been solved for the bubble radius increasing with time under a constant mean pressure [Skinner, 1972; Fyrillas and Szeri, 1994]. However, to the best of our knowledge, no solution has yet been presented for the geophysically important transient problem in which the average pressure increases with time while the average bubble radius does not change. In this paper we derive such a solution to show that the increase in background pressure limits the rate of mass transfer which in turn limits the total pressure rise. The mathematical technique in this paper is based on Fyrillas and Szeri [1994] (hereinafter referred to as FS).

[6] We first use the advective-diffusion equation in spherical symmetry to describe the transport of gas with the appropriate time-dependent boundary conditions. Then we transform the equation into a Lagrangian reference frame and split the system into smooth and oscillating parts. We then recognize that the smooth part is a diffusion problem with a small perturbation of the diffusion layer due to the oscillatory bubble motion. We derive a numerically tractable solution that describes the long-term pressure rise as an expansion in terms of the amplitude of the imposed strain. We ultimately show that the pressure rise from rectified diffusion is limited by the imposed strain regardless of the degree of supersaturation of the system.

\section{Formulation}

[7] The transport of dissolved gas around a bubble is described by an advection-diffusion equation in spherical coordinates and a flux boundary condition at the bubble wall.

$$
\frac{\partial C}{\partial T}+\frac{X^{2} \dot{X}}{R^{2}} \frac{\partial C}{\partial R}=\frac{D}{R^{2}} \frac{\partial}{\partial R}\left(R^{2} \frac{\partial C}{\partial R}\right)
$$




$$
\dot{M}_{g}=\left.4 \pi X^{2} D \frac{\partial C}{\partial R}\right|_{R=X} .
$$

where $T$ is the time, $R$ is the radial coordinate, $C(R, T)$ is the mass of the gas in a unit volume of solution, $X(T)$ is the bubble radius, $D$ is the diffusivity of the gas in the liquid, and $M_{g}(T)$ is the total mass of gas in the bubble. The superposed dot indicates $d / d T$.

[8] The gas inside the bubble is governed by an equation of state. We assume an isothermal ideal gas with

$$
P_{g} X^{3} / M_{g}=P_{g o} X_{o}^{3} / M_{g o} \text {. }
$$

where the subscript $o$ indicates initial values at time $T=0$. The ideal gas assumption is a satisfactory description of water behavior at magmatic temperatures and pressures up to at least $140 \mathrm{MPa}$ [Bowers, 1995].

[9] The boundary and initial conditions are,

$$
\begin{gathered}
C(R=X, T)=k P_{g}^{1 / 2} \sim 0.5 k P_{g o}^{1 / 2}\left(P_{g} / P_{g o}+1\right), \\
C(R \rightarrow \infty, T)=C(R, T=0)=C_{o}
\end{gathered}
$$

where $P_{g}(T)$ is the pressure of the gas in the bubble and $k$ is a constant. It is known as a good first approximation that water solubility of various magma is proportional to squareroot of the gas pressure. Equation (4) is the critical boundary condition for the mass transport problem. Since volatiles can be neither super- nor sub-saturated at the phase boundary, the concentration of the dissolved volatiles at the bubble wall must increase with the gas pressure in the bubble. For mathematical tractability, equation (4) is linearized around $P_{g}=P_{g o}$ without losing its essential role.

[10] The bubble boundary is constantly moving for an oscillating bubble, so we transform the coordinates to a Lagrangian reference frame in which the bubble wall is stationary. The transformation is

$$
\sigma=\left(R^{3}-X^{3}\right) /\left(3 X_{o}^{3}\right) .
$$

The other dimensionless variables, which are denoted by lower case letters, are defined as

$$
\begin{gathered}
t=T / T_{o}, c=\left(C-C_{o}\right) / \rho_{g o}, \\
x=X / X_{o}, p_{g}=P_{g} / P_{g o}, m_{g}=M_{g} / M_{g o},
\end{gathered}
$$

where $T_{o}$ is the period of the acoustic field, and $\rho_{g o}=M_{g o} /$ $\left(\frac{4}{3} \pi X_{o}^{3}\right)$. The initial concentration $C_{o}$ has been subtracted for convenience.

[11] The dimensionless equations and boundary conditions are now

$$
\begin{gathered}
\frac{\partial c}{\partial t}=\frac{1}{P e} \frac{\partial}{\partial \sigma}\left[\left(3 \sigma+x^{3}\right)^{\frac{4}{3}} \frac{\partial c}{\partial \sigma}\right], \\
\frac{d m_{g}}{d t}=\left.\frac{3}{P e} x^{4} \frac{\partial c}{\partial \sigma}\right|_{\sigma=0}, \\
p_{g} x^{3} / m_{g}=1, \\
c(\sigma=0, t)=c_{s b}\left(p_{g}(t)+1\right)-c_{o}, \\
c(\sigma \rightarrow \infty, t)=c(\sigma, t=0)=0 .
\end{gathered}
$$

Equations (8)-(12) have three dimensionless parameters. One is the Peclet number defined as $P e=\left(X_{o}^{2} / D\right) / T_{o}$, which is the ratio of the diffusive time scale and the oscillation period. The others are $c_{s b}=1 / 2 k P_{g o}^{1 / 2} / \rho_{g o}$ and $c_{o}=C_{o} / \rho_{g o}$. When the liquid and bubble are in equilibrium before oscillation starts, $c_{o}=2 c_{s b}$.

[12] FS solved equations (8)-(12) assuming that the pressure, $p_{g}$, is a prescribed periodic function. Here we use the same method except that we assume that a periodic volumetric strain from the seismic wave makes the bubble radius, $x$, follow a prescribed periodic function.

\section{Splitting of the Problem}

[13] We now split the problem into oscillatory and smooth parts in order to isolate the contribution to the long-term pressure rise. In order to perform the separation, we must first introduce the following two averaging procedures:

$$
\begin{gathered}
\langle f(t)\rangle=\frac{1}{t_{o}} \int_{n t_{o}}^{(n+1) t_{o}} f(t) d t, \\
\langle f(t)\rangle_{\hat{\tau}}=\frac{1}{\hat{\tau}\left(t_{o}\right)} \int_{\hat{\tau}\left(n t_{o}\right)}^{\hat{\tau}\left((n+1) t_{o}\right)} f(\hat{\tau}) d \hat{\tau} ; \hat{\tau}(t)=\int_{0}^{t} x^{4}(\theta) d \theta,
\end{gathered}
$$

where $f$ is an arbitrary function of $t, n$ is an integer and $t_{o}$ is the dimensionless period of bubble oscillation. (Although $t_{o}=1$ from the definition of dimensionless time, we retain the symbol $t_{o}$ to represent the cycle explicitly.) Equation (13) is an ordinary average over a cycle of the wave; equation (14) is an average over a cycle weighted by $x^{4}$. The weighting factor of $x^{4}$ is a manifestation of the two mechanisms that combine to generate rectified diffusion. One factor of $x^{2}$ is due to change of the bubble surface, and another $x^{2}$ is due to the sharpening of the radial gradient of the volatile concentration in the diffusion layer which is thinned by bubble expansion as $\propto x^{-2}$.

[14] Now we rewrite the boundary condition equation (11) by adding and subtracting the weighted average $\left\langle p_{g}(t)\right\rangle_{\hat{\tau}}$.

$$
\begin{aligned}
c(\sigma=0, t)= & c_{s b}\left[p_{g}(t)-\left\langle p_{g}(t)\right\rangle_{\hat{\tau}}\right] \\
& +\left[c_{s b}\left(\left\langle p_{g}(t)\right\rangle_{\hat{\tau}}+1\right)-c_{o}\right] .
\end{aligned}
$$

The first bracket in equation (15) with equations (8) and (12) determines the oscillatory part of the concentration, $c_{\text {osc }}(\sigma, \tau)$, while the second bracket with the same equations determines the smooth part of the concentration, $c_{s m}(\sigma, \tau)$. Although we followed FS to split the problem in this way, we have made one important change in equations (13) and (14). We treat the shift of the average pressure with increasing number of the oscillation cycles, while FS defined the integrals in a fixed range from $t=0$ to $t_{o}$.

[15] The second bracket on the right-hand side of equation (15) describes the acoustic equilibrium volatile concentration at which an oscillating bubble is stable, that is, the concentration at which the bubble neither resorbs nor grows [Eller and Flynn, 1965]. Rectified diffusion makes an oscillating bubble stable at a concentration below that of a stationary bubble. Because the volatile concentration around an oscillating bubble is more affected by the pressure during bubble expansion than that during contraction the average 
pressure weighted by the bubble radius determines the acoustic equilibrium condition rather than the ordinary mean pressure.

[16] The analytical solution of FS for the oscillatory problem is applicable to the present problem, because the oscillatory boundary condition is the same. The asymptotic form of $c_{\text {osc }}(\sigma, \tau)$ brings no mass transport over one period, and the mass transport associated with its transient is negligible. The smooth part causes mass transport into the bubble and hence increases pressure of the system. Therefore, we focus on the smooth problem.

\section{The Smooth Problem}

[17] The smooth problem is characterized by slow diffusion processes. Therefore, we introduce a second time scale, $\lambda=t / P e$, and distinguish it from the shorter time of the oscillation, $\tau=t$. Following FS and Hinch [1991], we transform the time-derivative into short and long-term components using

$$
\frac{\partial}{\partial t}=\left(\frac{\partial}{\partial \tau}\right)_{\lambda}+\frac{1}{P e}\left(\frac{\partial}{\partial \lambda}\right)_{\tau}
$$

[18] Like previous treatments of rectified diffusion, we assume that the Peclet number is large and so the variables can meaningfully be expanded with respect to $P e^{-1}$. The typical period range of the large amplitude shaking of large earthquakes is $1-20 \mathrm{~s}$. For magma of which water diffusivity is typically $D \leq 10^{-9} \mathrm{~m}^{2} / \mathrm{s}$ [Zhang and Stolper, 1991], $P e \gg 1$ if and only if the bubble radius is much larger than $100 \mu \mathrm{m}$. Such bubble radius is reasonable but not necessary inside a magma chamber according to a recent model for bubble nucleation in magma [Yamada et al., 2005].

[19] If the Peclet number is small, then rectified diffusion cannot work because the system can reequilibriate diffusively faster than the oscillations and there is no correction necessary to the ordinary diffusive effect. So, by limiting ourselves to large Peclet numbers (large bubbles), we study the regime in which rectified diffusion is most likely to be effective. If we show that it is a weak effect in this regime, then it is likely to be a weak effect overall.

[20] The smooth part of the concentration can be expanded as $c_{s m}(\sigma, t)=c_{s m}^{0}(\sigma, \lambda, \tau)+P e^{-1} c_{s m}^{1}(\sigma, \lambda, \tau)+\cdots$. FS show that the zeroth-order term of the expansion and the corresponding approximation of the rate of mass transport (9) are determined by

$$
\begin{gathered}
\frac{\partial c_{s m}^{0}}{\partial \tau}=0, \\
\frac{\partial c_{s m}^{0}}{\partial \lambda}=\frac{\partial}{\partial \sigma}\left(\left\langle\left(3 \sigma+x^{3}\right)^{\frac{4}{3}}\right\rangle \frac{\partial c_{s m}^{0}}{\partial \sigma}\right), \\
c_{s m}^{0}(\sigma=0, \lambda, \tau)=c_{s b}\left(\left\langle p_{g}\right\rangle_{\hat{\tau}}^{0}+1\right)-c_{o}, \\
c_{s m}^{0}(\sigma \rightarrow \infty, \lambda, \tau)=c_{s m}^{0}(\sigma, \lambda=0, \tau=0)=0, \\
\frac{d m_{g, s m}^{0}}{d \lambda}=\left.3\left\langle x^{4}\right\rangle \frac{\partial c_{s m}^{0}}{\partial \sigma}\right|_{\sigma=0} .
\end{gathered}
$$

Because we are considering strictly periodic change of the bubble radius, $\left\langle\left(3 \sigma+x^{3}\right)^{\frac{4}{3}}\right\rangle$ depends on neither $\tau$ nor $\lambda$. However, in the confined system case studied here, the weighted average pressure in equation (20) varies with an increasing number of cycles. This weighted average pressure is expanded with respect to $P e^{-1}$, and $\left\langle p_{g}\right\rangle_{\hat{\tau}}^{0}$ in equation (19) is its leading term.

[21] We may regard the average pressure in the bubble as functions of $\lambda$ and transform the integrals in equation (13) and (14) with $\int_{n t}{ }^{(n+1) t_{o}} d t \rightarrow \int_{\lambda}^{\lambda+t_{o}} d \tau$. Because $x$ and the oscillatory part of $m_{g}$ do not vary on the long timescale described by $\lambda$, and $m_{g, s m}$ does not depend on the short time scale, $\tau$, equation (10) results in

$$
\frac{d\left\langle p_{g}\right\rangle_{\hat{\tau}}}{d \lambda}=\frac{d}{d \lambda}\left\langle\frac{m_{g, s m}}{x^{3}}\right\rangle_{\hat{\tau}}=\frac{\langle x\rangle}{\left\langle x^{4}\right\rangle} \frac{d m_{g, s m}}{d \lambda}
$$

Substituting equation (21) into equation (22), we obtain the rate of the average pressure increase associated with the zeroth-order approximation of the smooth problem as

$$
\frac{d\left\langle p_{g}\right\rangle_{\hat{\tau}}^{0}}{d \lambda}=\left.3\langle x\rangle \frac{\partial c_{s m}^{0}}{\partial \sigma}\right|_{\sigma=0} .
$$

[22] The initial condition for $\left\langle p_{g}\right\rangle_{\hat{\tau}}^{0}$ is defined as the average with respect to $\hat{\tau}$ during the first period of bubble oscillation. We neglect the change of the mass within the bubble then, because the time is too short for mass transport associated with the smooth part to be effective and the amplitude of the mass transport in the oscillatory part is of order $P e^{-1 / 2}$ [FS]. Using the equation of state equation (10) with $m_{g}=1$ initially,

$$
\left\langle p_{g}\right\rangle_{\hat{\tau}}^{0}(\lambda=0)=\langle x\rangle /\left\langle x^{4}\right\rangle .
$$

[23] Now we have a set of equations for the zeroth-order approximation of the smooth problem: equations (18) and (23) with the boundary conditions (equations (19) and (20)), and the initial condition equation (24). FS solved the smooth problem analytically with a perturbation expansion with respect to small changes in bubble size. Although we have not been able to obtain an analytical solution for the present problem, we follow their mathematical procedure because it makes the contribution of bubble oscillation to the pressure increase clearer.

[24] We assume the volume changes is

$$
x^{3}(\tau)=1+\delta_{v} \sin \left(2 \pi \tau / t_{o}\right)
$$

where $\delta_{v} \ll 1$. In order to solve for terms like $\langle x\rangle$, we will expand equation (25) in powers of $\delta_{v}$. The first two leading terms of the expansion are $O(1)$ and $O\left(\delta_{v}^{2}\right)$, because $\delta_{v}$ $\sin \left(2 \pi \tau / t_{o}\right)$ vanishes by averaging over one cycle.

[25] We reach our final form of the equations by substituting in the volume oscillation of equation (25) into the combined equations (18), (19), (20), (23) and (24) rewritten with the variable transformations: $z=(3 \sigma+1)^{1 / 3}-1$ and $c_{s m}^{0}(\sigma, \lambda)=n(z, \lambda) /(z+1)$. The former transformation reverts the coordinate system to an Eulerian reference frame and the latter transforms the spherical diffusion equation to a one- 


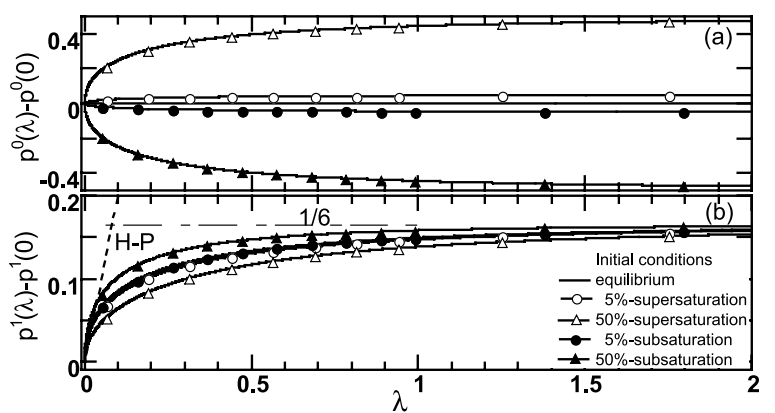

Figure 1. Numerical solutions for (a) $p^{0}$ and (b) $p^{1}$ for various initial conditions: equilibrium, supersaturated, and undersaturated. Since the expansion is in terms of $\delta_{v}{ }^{2}$, in the presence of a super- or subsaturation, the contribution of the $p^{1}$ term to the total pressure and mass change is significantly less than the zeroth order terms. The horizontal broken line in Figure $1 \mathrm{~b}$ indicates $1 / 6$, which is the maximum dimensionless pressure increase by the rectified diffusion. The dotted line labeled H-P is the Hsieh and Plesset [1961] solution.

dimensional one. Then the equations are solved using a perturbation in the small parameter $\delta_{v}^{2}$, of the form

$$
\begin{gathered}
n(z, \lambda)=n^{0}(z, \lambda)+\delta_{v}^{2} n^{1}(z, \lambda)+\cdots \\
\left\langle p_{g}\right\rangle_{\hat{\tau}}^{0}(\lambda)=p^{0}(\lambda)+\delta_{v}^{2} p^{1}(\lambda)+\cdots
\end{gathered}
$$

[26] The zeroth- and first-order problems are represented with $j=0,1$ as

$$
\begin{aligned}
& \frac{\partial n^{j}}{\partial \lambda}=\frac{\partial^{2} n^{j}}{\partial z^{2}}+\frac{j}{9(z+1)} \frac{\partial}{\partial z}\left[\frac{1}{(z+1)^{4}} \frac{\partial}{\partial z}\left(\frac{n^{j-1}}{z+1}\right)\right], \\
& \frac{d p^{j}}{d \lambda}=\left.3 \frac{\partial}{\partial z}\left(\frac{n^{j}}{z+1}\right)\right|_{z=0}-\left.\frac{j}{6} \frac{\partial}{\partial z}\left(\frac{n^{j-1}}{z+1}\right)\right|_{z=0} \\
& n^{0}(0, \lambda)=c_{s b}\left(p^{0}+1\right)-c_{o}, \quad n^{1}(0, \lambda)=c_{s b} p^{1}, \\
& n^{0}(\infty, \lambda)=0, \quad n^{1}(\infty, \lambda)=0, \\
& n^{0}(z, 0)=0, \\
& n^{1}(z, 0)=0, \\
& p^{0}(0)=1, \\
& p^{1}(0)=-1 / 6 \text {. }
\end{aligned}
$$

[27] The zeroth order problem includes no effect from bubble oscillation. If the initial condition is saturated, which corresponds to $2 c_{s b}=c_{o}$, equations (27) $-(29)$ with $j=0$ give $n^{0}(z, \lambda)=0$ and $p^{0}(\lambda)=1$. The effect of rectified diffusion appears in the first order problem. The non-zero initial condition for $p^{1}$ represents the difference between the nonlinear time average of the pressure, $\left\langle p_{g}\right\rangle_{\hat{\tau}}$, and the static pressure, $p^{0}$. This difference is the reason that, even in the initially saturated case, net mass transport occurs during oscillation [Eller and Flynn, 1965; FS]. In case of an initially non-equilibrium condition, $n^{0}$ is non-zero, which is not due to rectified diffusion but due to ordinary diffusion.
[28] The first-order equations include $n^{0}$ as source terms, indicating that the rectified diffusion and the ordinary diffusion are coupled (equations (27) and (28) with $j=1$ ). However, this effect is shown to be small by numerical calculations.

\section{Numerical Results}

[29] Equation (27)-(29) are solved numerically. The differential equations in space are discretized using a finite difference method of the second degree of accuracy. Integration with respect to time is done implicitly.

[30] Figure 1 shows the changes of pressure from ordinary diffusion, $p^{0}(\lambda)-p^{0}(0)$, and rectified diffusion, $p^{1}(\lambda)-p^{1}(0)$ as a function of the slow timescale $\lambda$. We give results for various initial conditions including equilibrium $\left(c_{s b}\left(p^{0}+1\right)=c_{o}\right)$, supersaturated $\left(c_{s b}\left(p^{0}+1\right)<c_{o}\right)$, and undersaturated $\left(c_{s b}\left(p^{0}+1\right)>c_{o}\right)$ cases. The zeroth-order curves (Figure 1a) represent the change due to ordinary diffusion which occurs regardless of bubble oscillation if a non-equilibrium initial condition is given. The first-order curves for all the cases approach an identical value (Figure 1b). Because the mass transport stops when the acoustical equilibrium condition is achieved, the contribution of rectified diffusion is bounded by $\delta^{2}{ }_{v} / 6$ of the initial pressure regardless of initial saturation conditions. Moreover, both the ordinary and rectified diffusion are controlled by the time scale of volatile diffusion in the liquid, so the former cannot significantly accelerate the latter.

[31] We compare our result with that obtained by Hsieh and Plesset [1961] who derived the mass transport for the unconfined case (Figure 1b). The solution of Hsieh and Plesset [1961] is represented in a dimensionless form using the same scales in the previous section as:

$$
d m_{g, H P} / d \lambda=2 \delta_{v}^{2}
$$

Equation (22) is integrated with respect to $\lambda$ using equation (30) and the initial condition for $\left\langle p_{g}\right\rangle_{\hat{\tau}}^{0}$ in equation (29). We then have

$$
\left\langle p_{g}\right\rangle_{\hat{\tau}, H P}^{0}=1+\delta_{v}^{2}(2 \lambda-1 / 6)+\mathcal{O}\left(\delta_{v}^{4}\right)
$$

We compare equation (31) and our numerical solution for $p^{1}$ in Figure 1b. It is obvious that the solution of Hsieh and Plesset [1961] becomes significantly larger than the present one with time.

[32] Because the mass transport into the bubble is suppressed by the pressure increase in a fixed volume, the change of the mass due to rectified diffusion will never exceed $O\left(\delta_{v}^{2}\right)$, and neither will the pressure increase. Typical seismic waves from magnitude $7-8$ earthquakes at $100 \mathrm{~km}$ have strain amplitudes $\sim 10^{-4}-10^{-5}$ [Brodsky et al., 1998]. The waves can increase the pressure by no more than $2 \times 10^{-9}$ of its initial pressure.

\section{Conclusion}

[33] The effect of rectified diffusion increasing magma pressure as a result of a strain wave from a distant earthquake has been re-evaluated by incorporating the effect of pressure increase on solubility in a confined system. 
Rectified diffusion by itself can cause only a slight pressure increase. The limiting value of the pressure increase is determined entirely by the imposed volumetric strain. For reasonable seismic wave amplitudes, the pressure change is $<2 \times 10^{-9}$ of its initial value. Rectified diffusion may yet play a role in water- $\mathrm{CO}_{2}$ system due to combination with evaporation [Kawashima et al., 2001], but our calculations show that as an isolated mechanism it is incapable of generating significant pressure from realistic seismic wave amplitudes.

[34] Acknowledgments. This work was stimulated by conversations with the late B. Sturtevant. We thank H. Huang, H. Kanamori, F. Nimmo, S. Prejean, K. Felzer, and S. Takagi for helpful suggestions to improve the manuscript.

\section{References}

Bowers, T. S. (1995), Pressure-volume-temperature properties of $\mathrm{H}_{2} \mathrm{O}-\mathrm{CO}_{2}$ fluids, in Rock Physics and Phase Relations: A Handbook of Physical Constants, AGU Ref. Shelf, vol. 3, edited by T. J. Ahrens, pp. 45-72, AGU, Washington, D. C.

Brodsky, E. E., B. Sturtevant, and H. Kanamori (1998), Earthquakes, volcanoes, and rectified diffusion, J. Geophys. Res., 103, 23,827-23,838.

Eller, A., and H. G. Flynn (1965), Rectified diffusion during nonlinear pulsations of cavitation bubbles, J. Acoust. Soc. Am., 37, 493-503.
Fyrillas, M. M., and A. J. Szeri (1994), Dissolution or growth of soluble spherical oscillating bubbles, J. Fluid Mech., 277, 381-407.

Hinch, E. J. (1991), Perturbation Methods, 160 pp., Cambridge Univ. Press, New York.

Hsieh, D.-Y., and M. S. Plesset (1961), Theory of rectified diffusion of mass into gas bubbles, J. Acoust. Soc. Am., 33, 206-215.

Kawashima, H., M. Ichihara, and M. Kameda (2001), Dynamics of spherical vapor/gas bubble in acoustic fields, paper presented at 4th International Conference on Multiphase Flow, Gov. Board of the ICMF, New Orleans, LA.

Linde, A. T., and I. S. Sacks (1998), Triggering of volcanic eruptions, Nature, 395, 888-890.

Skinner, L. A. (1972), Acoustically induced gas bubble growth, J. Acoust. Soc. Am., 51, 378-382.

Sturtevant, B., H. Kanamori, and E. E. Brodsky (1996), Seismic triggering by rectified diffusion in geothermal systems, J. Geophys. Res., 101, $25,269-25,282$

Yamada, K., H. Tanaka, K. Nakazawa, and H. Emori (2005), A new theory of bubble formation in magma, J. Geophys. Res., 110, B02203, doi:10.1029/2004JB003113.

Zhang, Y. X., and E. M. Stolper (1991), Water diffusion in a basaltic melt, Nature, 351, 306-309.

E. Brodsky, Department of Earth and Space Sciences, UCLA, Los Angeles, CA 90095, USA. (brodsky@ess.ucla.edu)

M. Ichihara, Earthquake Research Institute, University of Tokyo, Yayoi 1-1-1, Bunkyo-ku, Tokyo 113-0032, Japan. (ichihara@eri.u-tokyo.ac.jp) 